\title{
Una experiencia de economía solidaria
}

\author{
Sofía Cerón, laura Mejía, Miguel Parada, \\ Enrique Moreno y Juan Manuel Zavaleta ${ }^{1}$
}

\section{Introducción}

El Departamento de La Paz, lugar donde se encuentra el municipio de Zacatecoluca, fue uno de los más afectados por los terremotos ocurridos en los meses de enero y febrero de 2001. También hay que señalar que este departamento sufrió el paso del huracán Mitch en 1998.

Las consecuencias de estos acontecimientos agravaron las condiciones de vida de la población más pobre de El Salvador. Por ejemplo, en las comunidades de Zacatecoluca identificadas por Solidaridad Internacional Andalucía el $50 \%$ de las viviendas se vieron destruidas y la mitad un $40 \%$ de ellas quedaron muy dañadas. La totalidad de las letrinas de fosa se hundieron, contaminando el acuífero; todos los pozos de agua superficial también se contaminaron y sus paredes se vinieron abajo al carecer de recubrimiento.

Bajo este contexto, nosotros hemos centrado el estudio en la comunidad San José las Flores, ubicada en el municipio de Zacatecoluca, Departamento de la Paz. Las Unidades Domésticas (UD) en la Comunidad son las 79 familias habitantes.

En respuesta a la situación que trágicamente vivieron los habitantes de la Comunidad San José las Flores, existen ciertas Organizacio- 
nes No Gubernamentales (ONG) que, históricamente, han apoyado a la comunidad con el recurso humano y económico.

Las Asociaciones Voluntarias que apoyan a la fecha a la comunidad, son todas Organizaciones No Gubernamentales para el Desarrollo (ONGD): Fundación Nacional para el Desarrollo (FUNDESA), Asociación Humanitaria de Apoyo Provida y Solidaridad Internacional de Andalucía. Estas ONGD trabajan en la prevención y mitigación, a través de proyectos de desarrollo, y en la atención de desastres, a través de campañas de ayuda de emergencia. Sin embargo, no han limitado su trabajo a esta última, la cual en determinadas situaciones y momentos es de gran importancia, sino que continuando con la labor ya realizada en esta comunidad, se pusieron en marcha nuevos proyectos, con el fin de iniciar en la zona un proceso de desarrollo a largo plazo.

Con este objetivo de cooperación al desarrollo, y de reducir el estado de vulnerabilidad de esta comunidad, Solidaridad Internacional Andalucía con la colaboración de las organizaciones locales, FUNDESA y Provida, lleva cabo ciertos programas en Zacatecoluca, donde desarrollan actividades en diferentes sectores:

- Infraestructura básica y comunal (dispensario medico, casa comunal multiusos, acceso a red eléctrica, construcción de viviendas),

- Producción agropecuaria (parcelas agroforestales, cría de aves, camas de siembra, aboneras, sistema de micro riego, asistencia técnica)

- Acceso a la salud y educación sanitaria (diagnóstico nutricional, campañas de salud, equipamiento y puesta en marcha del dispensario médico).

Gracias a todas estas actividades, la realidad social y económica de la comunidad se ha transformado: se ha reducido a cero las familias sin vivienda digna en la Comunidad San José Las Flores, ha aumentado la capacidad productiva de la Comunidad y se ha reducido la incidencia de enfermedades que afectaban especialmente a mujeres, niños $\mathrm{y}$ ancianos.

La comunidad San José las Flores es una economía solidaria y su forma de organización, reproducción y estilos de vida concuerdan con el concepto de Economía Popular Solidaria. 
Existen algunas formas de asociatividad de los habitantes, quienes se organizan con la finalidad de acceder a servicios comunes, para lograr dialogar y/o coordinar con el Estado (central o local) para obtener normas favorables para su desarrollo o bien para concertar en función del desarrollo local.

La Comunidad interactúa con otras comunidades aledañas y existen también algunas Redes de Ayuda Mutua, a través de intercambios mercantiles o de reciprocidad en los proyectos internos.

El trabajo de esta economía se funda en que las Unidades Domésticas (las familias) trabajan conjuntamente con las Comunidades de la región y las redes de ayuda mutua (Solidaridad Internacional de Andalucía, FUNDESA y Provida), en el acompañamiento del desarrollo regional de la comunidad a través de una serie de programas (detallados más adelante) que buscan ser solamente la base. No se trata de una erradicación simple de los problemas focales, sino más bien de una erradicación desde la raíz, generando fuentes de empleo e ingresos desde y para los habitantes de la comunidad. Así, el desarrollo sustentable dependerá y quedará en manos de los mismos habitantes y su capacidad y esfuerzo de progreso.

A lo largo del trabajo presentaremos un diagnóstico detallado de lo presenciado en las visitas a la comunidad que está avanzando hacia un tipo de economía solidaria, aún no desarrollada completamente.

\section{Marco teórico}

El campo económico está hoy conformado por ${ }^{2}$ la combinación de:

1. Una economía capitalista, monopolizada y regida por la acumulación acelerada de ganancias sin límites. Se organiza mediante empresas, asociaciones y Redes de empresas articuladas por la propiedad o grupos de control, o por el juego de relaciones de mercado.

2. Una economía pública, bajo reforma estructural, regida por la acumulación de poder político y los requisitos de gobernabilidad del sistema (o la construcción democrática de un interés general) - que combinan funciones reguladoras mínimas con políticas sociales asistencialistas-. Se organiza mediante los sistemas administrativoburocráticos bajo normas legales y prácticas de gestión y control administrativo-político. 
3. Y ese conjunto que denominamos economía solidaria, regido por la reproducción biológica y social de sus miembros. Esta economía será la base de nuestro estudio y por tanto se detalla más adelante.

La perspectiva de la economía del trabajo ${ }^{3}$ propone que la racionalidad económica de los pobres se expresa en la lógica del trabajo. Una pregunta relevante es por qué la mayoría de las actividades de autoempleo y microempresa en los sectores populares y campesinos de un país pobre como el nuestro, pese a que no logran cubrir sus costos reales, sin embargo siguen funcionando. Si la lógica de la maximización de la ganancia fuera el factor explicativo de sus economías, por lo menos el $90 \%$ de ellas ya deberían haber dejado de funcionar. La respuesta sencilla es que es su medio de vida.

A partir de las experiencias de pobladores de la ciudad y el campo en su lucha por sobrevivir y mejorar sus condiciones de vida, podemos sintetizar el concepto de economía popular como el conjunto de actividades de producción, distribución o consumo que realizan estas personas y organizaciones, para generar ingresos, empleo o para reducir los costos de vida accediendo a bienes básicos (alimentos, medicinas, vivienda, servicios básicos, etc).

Concebir la economía local como una estructura de tres subsistemas (la economía popular, la economía pública y la economía capitalista) permite pensar en la necesidad de incidir sobre los términos del intercambio entre la economía popular y la economía pública, o entre la economía popular y la economía capitalista y no sólo sobre el salario real que es sólo uno de los precios que afecta a la economía popular. Entre esos subsistemas se dan intercambios de bienes materiales, de servicios, de información, y transferencias (como los subsidios o los impuestos), cuyo balance agregado puede ser objeto de negociación social y política, o de modificación mediante el ejercicio del poder económico o político de los sectores populares.

Antes se pensaba que dentro de las naciones del Tercer Mundo los pobres eran importantes, porque los "movimientos populares" constituían una fuerza y una amenaza real al sistema establecido. Actualmente el mundo de los pobres ha perdido gran parte de su fuerza, su organización y su capacidad de lucha. Los pobres no tienen fuerza social y política. Por tanto, el que se pone al lado de los pobres no obtiene ventajas, ni siquiera reconocimiento genuino. Hace algunas 
décadas hacer una "opción por los pobres" constituía para muchos un motivo de orgullo. Si en la década de los sesenta la pobreza y la liberación de los pobres era el gran tema de los sociólogos e intelectuales, hoy lo que interesa es la modernidad y el paso a la postmodernidad. Ya no la transformación, sino la globalización.

Los gobiernos y los partidos políticos hablan todavía de la pobreza y afirman la gran mayoría de ellos, que es su gran preocupación. Pero no actúan consecuentemente con el interés manifestado. En realidad, la principal preocupación es mantenerse vinculados a los mercados internacionales y sus sofisticadas dinámicas: la revolución de la informática, la bioingeniería, las nuevas tecnologías, el consumo sofisticado, los nuevos instrumentos de la especulación financiera, etc.

Aunque se encuentre concentrada y segregada territorialmente, y aunque haya perdido gran parte de su potencial de lucha y acción, la pobreza continúa presentándose como un peligro para el resto de la sociedad. Por un lado, la pobreza crea inseguridad ciudadana, porque las expectativas de ocupación y de ingresos mínimos, sin esperanzas de progreso e integración normales, generan comportamientos y actitudes de rechazo y rebeldía social, expresados a menudo por medios violentos y antisociales que atentan contra las personas y la propiedad.

Por otro lado, la pobreza contamina y amenaza el equilibrio del medio ambiente. La pobreza agrava el problema ecológico. Una población extremadamente pobre concentrada en zonas densamente pobladas de inestable urbanización, carece de medios para cuidar y limpiar su medio ambiente inmediato. Por ejemplo: el efecto negativo del polvo que se levanta en calles sin pavimentar, el humo que libera la combustión de la madera, los desechos y basuras que no obtienen adecuada canalización, se expande por la atmósfera y las aguas contaminando la ciudad y su entorno agrícola, con consecuencias muy serias para la salud de toda la población.

Lo cual hace volver la vista a los pobres, con la intención de defender el propio bienestar alcanzado y no por una sincera preocupación por ellos. La respuesta tiende a ser en gran medida represiva: fortaleciendo las fronteras que separan la pobreza del resto de la sociedad.

Sin embargo, no todas las alternativas experimentadas por la situación de pobreza tienen connotación negativa. Para ello, es preciso

\section{9}

Una experiencia de economia solidaria 
observar no solamente lo que le pasa a los pobres, sino especialmente lo que ellos bacen para enfrentar sus necesidades y problemas.

La acción que se despliega actualmente en los sectores populares pobres es diferente a la que se verificaba en el pasado, debido a las nuevas circunstancias y condiciones, la orientación principal que manifiestan ahora los esfuerzos de integración y superación de su propia pobreza, es en un plano directamente económico: la formación y establecimiento de numerosas pequeñas actividades productivas y comerciales cuyos protagonistas son los grupos sociales empobrecidos de los barrios y poblaciones marginales o rurales.

Para referirse a este fenómeno, economistas y sociólogos han acuñado diferentes expresiones: economía informal, pequeña producción popular urbana, economía sumergida, economía invisible, economía de solidaridad, economía de subsistencia, economía popular. No siempre estos diferentes términos aluden exactamente al mismo fenómeno pues establecen algunas diferencias en la realidad que identifican. Pero todos ellos engloban un universo de iniciativas y experiencias que incluye, al menos, lo siguiente ${ }^{4}$ : el trabajo mercantil o de reproducción, las microempresas familiares y las organizaciones económicas populares.

La economía popular se organiza mediante ${ }^{5}$ unidades domésticas (UD), redes de ayuda mutua, comunidades y asociaciones voluntarias diversas y a través de intercambios mercantiles o de reciprocidad.

Las economías domésticas (generalmente sobre la base de las familias) son la unidad elemental de constitución de este sistema, pero sus redes interactivas, de circulación de bienes, servicios e información, potenciadas por los centros que las apoyan, les dan organicidad. Para entender el funcionamiento de una economía popular, es importante conocer qué entendemos por UD. Cada UD es un grupo de individuos, que son cotidianamente responsables de la obtención (mediante su trabajo presente o mediante transferencias o donaciones de bienes, servicios o dinero) y distribución de las condiciones materiales necesarias para la reproducción inmediata de todos sus miembros. Una UD puede abarcar o articular uno o más hogares ${ }^{6}$, co-residentes o no, basados en la familia o no, y participar en una o más redes contingentes comunitarias (de reciprocidad) o públicas (de redistribución social) presentes en la sociedad local. 
El fondo de trabajo de una UD es el conjunto de capacidades de trabajo que pueden ejercer en condiciones normales los miembros hábiles de la misma para resolver solidariamente su reproducción. La realización de dicho fondo abarca sintéticamente las siguientes formas:

\section{Trabajo mercantil:}

- Trabajo por cuenta propia -individual o colectivo (por su pequeña se le denomina usualmente "micro emprendimiento mercantil"') productor de bienes y servicios para su venta en el mercado.

- Trabajo asalariado, vendido a empresas capitalistas, al sector público u a otras organizaciones o unidades domésticas; trabajo de reproducción propiamente dicha.

- Trabajo de producción de bienes y servicios para el autoconsumo de la UD.

- Trabajo de producción solidaria de bienes y servicios para el consumo conjunto de una comunidad.

- Trabajo de formación y capacitación.

Ha surgido desde la realidad de la pobreza la economía popular, que constituye un verdadero proceso de activación y movilización económica del mundo popular. Cuando la economía popular pone al centro apoyo mutuo, la cooperación y diversas formas de compartir (conocimientos, mercados, tecnología) entre sí y crecientemente con otros más pobres, la llaman algunos economistas «economía popular solidaria»; en este caso estas personas cultivan y desarrollan, a diversos grados, el valor ético de la solidaridad. En sus varias manifestaciones y formas, esta economía popular contiene importantes elementos de solidaridad que es importante reconocer y destacar ${ }^{8}$.

La cultura de los grupos sociales más pobres es naturalmente más solidaria que la de los grupos sociales de mayores ingresos. La experiencia de la pobreza, de la necesidad experimentada como urgencia cotidiana de asegurar la subsistencia, lleva a muchos a vivenciar la importancia de compartir lo poco que se tiene, de formar comunidades y grupos de ayuda mutua y de recíproca protección. Además cada persona o familia, al disponer de tan escasos recursos para realizar sus actividades económicas, necesita de los individuos cercanos que en- 
frentan igual necesidad para complementar la dotación mínima de factores indispensable para crear la pequeña unidad económica que le permita una operación viable.

En términos económicos, la solidaridad de estos sectores se manifiesta en la forma de donaciones y ha dado lugar a numerosas organizaciones sin fines de lucro, que canalizan, distribuyen, intermedian y ejecutan donaciones, con beneficios para terceros y no para ellas mismas, lo que conlleva a la formación de circuitos de producción y distribución de bienes y servicios que pueden ser considerados como una economía de donaciones institucionales. Cada institución que intermedia donaciones puede considerarse como una unidad económica que forma parte de la economía de solidaridad y que tiene gran relevancia para el desarrollo de ésta.

Otro aspecto importante de este tipo de economías es como desde situaciones y vivencias de marginación y extrañamiento emergen constantemente iniciativas tendientes a motivar, promover y efectuar la participación social en diferentes niveles, dando lugar a organizaciones sociales que adoptan los más variados tipos y modos de funcionamiento. Esta participación configura sujetos comunitarios, lo cual implica un proceso de constante comunicación, de intercambio de experiencias y de informaciones, de buscar el consenso a través de la puesta en común de los objetivos, ideas, intereses y aspiraciones de cada uno.

En las economías populares existe conciencia social, que se expresa en la acción o la lucha por el cambio de las estructuras sociales. Las motivaciones que generan energías transformadoras encuentran en ella cabida y oportunidades de superación y participación de los sectores sociales marginados en el orden económico y social establecido, y en ella pueden entregar todo su aporte creativo, para salir adelante.

El desarrollo económico, tal como se ha dado en el mundo moderno, parece haber llegado a niveles insostenibles, los cuales comienza a generar más problemas que beneficios: desequilibrios ecológicos, desintegración social, deterioro tendencial de la calidad de vida, pérdida del sentido humano del proceso, entre otros.

Un desarrollo alternativo ha de implicar el desarrollo de los sectores sociales menos desarrollados económicamente, que ha de ser ecológicamente sustentable, debe conducir a niveles superiores de in- 
tegración social, y ha de estar presidido por valores de justicia y solidaridad.

El campo de la economía en sí es, en esencia, un proceso de intercambio vital entre el hombre y la naturaleza, por el cual ambos resultan transformados. Pero los deterioros del medio ambiente nos hacen descubrir este proceso no siempre resulta positivo, pudiendo al contrario provocar desequilibrios que afectan al hombre mismo y que podrían incluso destruir la habitabilidad de la tierra.

Como la ecología depende de la economía, la existencia de un serio problema ecológico pone de manifiesto la existencia de muy serios problemas en la economía tal como se encuentra organizada actualmente, al tiempo que plantea la necesidad y urgencia de desarrollar otros modos de organizarla. Las causas económicas del deterioro ecológico se encuentran en el modo individualista, concentrador y excluyente, de una economía muy poco solidaria, que no se hace cargo de graves efectos sociales y medioambientales. Cuando se introduce la solidaridad en la economía, parece que las actividades económicas se tornan sanas, desde el punto de vista ecológico.

Otra vertiente desde donde surge la economía popular, es desde la realidad de la familia en crisis y desde la situación de la mujer, es ahí donde surge la posibilidad de un proceso de recuperación de personalidad y comunidad a la vez, impulsando a ciertos grupos de personas a experimentar otras formas de trabajo, producción y consumo. En el marco de la economía de solidaridad se torna posible crear condiciones para una recuperación de la familia como unidad social que realiza su verdadera vocación y plenitud de sentido, y para una nueva inserción de la mujer, no subordinada ni discriminatoria, en el trabajo y la sociedad.

Se trata, pues, de un desarrollo humano sustentable, para cuya promoción se hace imprescindible vincular el objetivo de largo plazo con la resolución inmediata de las necesidades, con un desarrollo visible de la calidad de vida, y todo eso tiene un componente económico. Pero aunque la centralidad de lo económico en esta coyuntura es importante, el desarrollo de la economía popular - que es un sistema de economía del trabajo, pero también de vinculación, de comunicación, de representación- requiere un enfoque integral, no economicista ${ }^{9}$. 
Pareciera que el planteamiento de este nuevo tipo de economía es idealista y utópico; que la realidad de esas diferentes experiencias no es tan solidaria como se dice o se quiere creer; que son todas experiencias pequeñas y casi siempre marginales. Pero no estamos hablando de metas logradas ni de realidades perfectas, sino de caminos, de iniciativas, de experiencias, de proyectos. Consiste, en verdad, en un proceso incipiente pero real, cuyos caminos recién empiezan a ser recorridos pero que muestran ya numerosas realizaciones y logros.

\section{Metodología de la investigación}

\subsection{Itinerario}

La investigación se desarrolló del $1^{\circ}$ de mayo hasta el 12 de junio, con etapas que alternaban visitas al terreno, investigación bibliográfica, entrevistas, períodos de análisis y de restitución.

El equipo visitó la comunidad San José las Flores, ubicada en el municipio de Zacatecoluca.

\subsection{Personas encuestadas}

Al visitar la comunidad el equipo intentó entrevistar un amplio abanico de interlocutores: población de la comunidad, representantes de asociaciones locales formales e informales (ADESCOS) y representantes de FUNDESA.

\subsection{Técnica de encuesta y restitución ${ }^{10}$.}

El equipo realizó observaciones directas, mediante visitas a las comunidades. En cada visita se buscó representantes de los diversos grupos de la población descritos anteriormente. Algunos de estos encuentros fueron preparados, otros se organizaron de manera espontánea (entrevistas semi-estructuradas). La mayoría de las entrevistas fueron individuales, pasando un total de 50 encuestas, por cuestiones ajenas al equipo de trabajo, que no obstante sigue siendo una muestra bastante representativa. Por otro lado también pudimos interactuar con los representantes de la Junta Directiva, mediante varios encuentros formales e informales.

El equipo alternó actividades de recolección de datos y períodos de análisis. Este documento incluye un diagnóstico de la comunidad, 
una vista a la comunidad como economía popular, un análisis de la implementación de los programas por parte de FUNDESA y algunas recomendaciones y conclusiones.

\section{Resultados de la investigación}

\subsection{Historia de la comunidad}

El 27 de noviembre de 1986, a consecuencia del conflicto armado, en Chalatenango se organizan 75 familias para formar una Cooperativa de la Reforma Agraria, programa implementado por el Gobierno del Presidente José Napoleón Duarte. Fue cuando, en los terrenos de la cooperativa Escuintla se segmentaron 436.5 metros de tierra. El 16 de marzo de 1987 se obtuvo la personería jurídica como Cooperativa de la Reforma Agraria, llamándose Asociación Cooperativa de la Reforma Agraria San José las Flores. Se llamó así, porque la mayoría de las 75 familias son originarias de San José las Flores Chalatenango.

En 1988 el señor Frankingler, procedente de Austria, hace la donación de la escuela y el puente de la comunidad. Este último se construyó sobre el río Amayo, pero en el año de 1998 cuando El Salvador se vio azotado por la tormenta tropical Mitch, generó grandes impactos en la comunidad, destruyéndolo totalmente y dejando sin acceso la salida hacia Zacatecoluca, como también ocasionando daños y pérdidas de cultivos y animales.

En noviembre del 2000, con el apoyo de FUNDESA, la comunidad comienza a organizarse como Asociación de Desarrollo Comunal (ADESCO), obteniendo la Personería Jurídica, en Marzo del 2001 de Asociación de Desarrollo Comunal San José las Flores (ADESCOSJF).

Con los terremotos del 13 de enero y febrero de 2001 la comunidad quedó totalmente destruida ya que todas las viviendas eran de adobe, un aproximado de 70 familias quedaron sin casas, la escuela también sufrió algunos daños. La primera ONG internacional que apoyó a la comunidad fue CHF Internacional-El Salvador, quien financió la reconstrucción de la escuela. Luego, la Junta Directiva comienza a hacer gestión para reconstruir la comunidad, y a través de FUNDESA y CARE El Salvador se construye y se benefician sesenta familias con viviendas provisionales. 
Más adelante, en el mismo año, a través de FUNDESA y la cooperación de Solidaridad Internacional de España se reconstruye la comunidad con 60 viviendas dignas permanentes, beneficiando a igual numero de familias, también se construyeron 2 pozos comunitarios para el consumo de agua, debido a los altos niveles de contaminación.

Adicionalmente y como parte de los proyectos de FUNDESA en el 2002, se lleva a cabo el proyecto de los módulos de agua y saneamiento en algunas viviendas que consiste en mejoramiento de pozos con tubos de concreto, bomba de mecate, un baño, una pila, lavadero, canaleta y fosa resumidero de aguas grises y la construcción de cuatro viviendas, lo que hace un total de 64 .

En el año 2003, nuevamente a través de FUNDESA y Solidaridad Internacional, se ejecutaron los proyectos de diez viviendas, módulos de producción integral (parcelas agroforestales, hortalizas, mini granjas de aves ponedoras, cocinas ahorradoras de leña), y la construcción de una clínica médica.

Existían para entonces 74 viviendas, pero, más adelante, como proyecto propio y con los recursos de ocho familias de la comunidad, ellas mismas construyeron sus viviendas. Esto hace un total de 82 viviendas.

En el mismo año FUNDESA Y CONCERN de Irlanda llevaron a cabo el proyecto Desarrollo Comunitario Rural que consistía en fortalecer la organización social y productiva como también capacitarlos para desarrollar nuevas habilidades y generar movilización e incidencia y participar en los cambios sociales, económicos y políticos de la comunidad y nuestro país.

Actualmente a través de FUNDESA y Solidaridad Internacional se encuentra en auto- construcción la casa comunal de usos múltiples, una red hidráulica de 7 cantareras y la iglesia católica.

\subsection{Datos generales}

\subsubsection{Población total y sexo}

El total de población de la comunidad San José las Flores está conformada de la siguiente forma: 


\section{Cuadro $\mathrm{N}^{\circ} 1$}

\begin{tabular}{|l|c|c|c|c|c|}
\hline $\begin{array}{l}\text { Niños } \\
\text { 0 a } 12 \text { años }\end{array}$ & $\begin{array}{l}\text { Jóvenes } \\
13 \text { a } 17 \text { años }\end{array}$ & Hombres & Mujeres & Familias & $\begin{array}{c}\text { Total } \\
\text { de la } \\
\text { Población }\end{array}$ \\
\hline 170 & 34 & 89 & 82 & 79 & 375 \\
\hline
\end{tabular}

Como podemos observar estos datos, se reflejan también en nuestra muestra (Gráfico $\mathrm{N}^{\circ} 3$ ) ya que el mayor porcentaje lo representan los hombres, a pesar de ello la diferencia en cuanto a las mujeres no es tan significativa. Con lo que podremos decir que el porcentaje de hombres y mujeres de la comunidad está casi por igual.

\section{Gráfico $N^{\circ} 1$}

\section{Sexo}
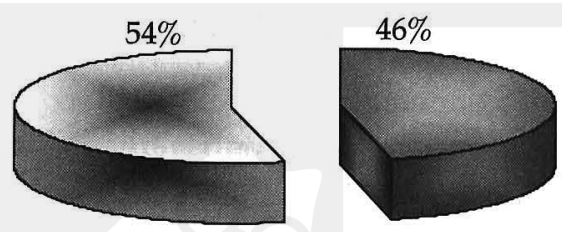

\section{圈Femenino}

aㅗ Mascullino

\subsubsection{Estado Familiar}

Dentro de las 79 familias de la comunidad, encontramos que si bien existen familias integradas por padre y madre. El 52\% respondió estar solamente acompañados/as (Gráfico $\mathrm{N}^{\circ} 2$ ), en contraposición con el $28 \%$, que está casadocasado. Un dato interesante es que ninguno respondió ser divorciado y tan solo una persona es viuda.

\section{Gráfico $\mathbf{N}^{\circ} 2$}

Estado familiar

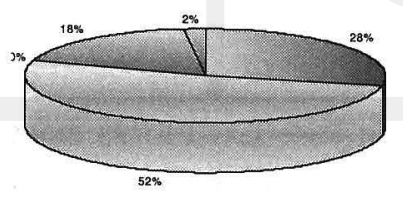

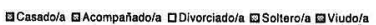




\subsubsection{Edad}

En el gráfico $\mathrm{N}^{\circ} 3$ se presentan las edades de las personas que respondieron a las encuestas, no las edades de la población en general, como se muestra en el cuadro $\mathrm{N}^{\circ} 1$. En

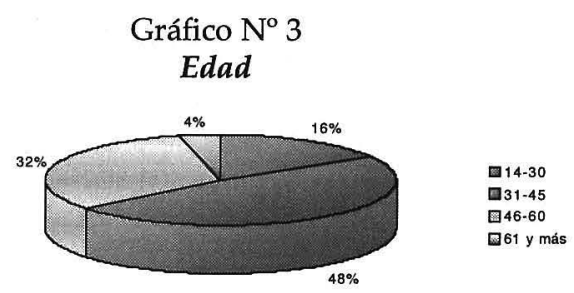
este estudio hay que tomar en cuenta que a través de los datos recolectados por medio de encuestas, el $80 \%$ de las personas que respondieron son personas adultas, que oscila entre las edades de 31 a 60 años. Estos datos verifican que las respuestas encontradas con respecto a la comunidad son bastante consistentes.

\subsubsection{Natalidad}

Un dato que no hay que dejar de lado, es el número de hijos que posee cada familia. Esto se ve reflejado en el aspecto de natalidad, el cual es bastante considerable en la comunidad.

Existen familias que tienen de 1 a 2 niños, pero también hay casos extremos en que cada familia de 12 a 15 hijos. Sin embargo, en el gráfico $\mathrm{N}^{\circ} 4$ vemos que solo el $12 \%$ tiene más de 7 hijos. La generali-

Gráfico $\mathrm{N}^{\circ} 4$

Número de hijos/as por familia
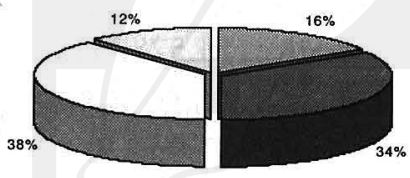
dad es que tengan de 3 a 6 hijos por familia. Respecto a este tema Provida ha impartido charlas por medio de educación popular, acerca del tema de sobrepoblación y métodos preventivos para el embarazo. Situación que nos parece bastante interesante, ya que a pesar de ser una comunidad rural con una educación bastante regular, estas charlas son bien recibidas sobre todo por las mujeres, aunque siempre existen aquellos que se rehúsan a tomarlas en cuenta.

\subsubsection{Educación}

En la comunidad San José Las Flores existe solamente una Escuela Pública (EDUCO) llamada "Centro de Estudios Caserío San José Las Flores" 
Cuadro $\mathrm{N}^{\circ} 2$

\begin{tabular}{|l|l|c|c|}
\hline Nombre de la Escuela & Ubicación & $\mathbf{N}^{\circ}$ Aulas & $\mathbf{N}^{\circ}$ Maestros \\
\hline $\begin{array}{l}\text { Centro Escolar } \\
\text { Caserío San José } \\
\text { las Flores }\end{array}$ & $\begin{array}{l}\text { Cantón } \\
\text { Tierra Blanca, } \\
\text { Zacatecoluca, } \\
\text { La Paz }\end{array}$ & 4 & 2 \\
\hline
\end{tabular}

La escuela da una cobertura desde parvularia hasta sexto grado, con un número actual de 120 alumnos, que se distribuyen en 4 aulas, lo que da un promedio de 30 alumnos por aula. Algo preocupante es que estos 120 alumnos reciben solamente la atención de 2 maestros.

En la comunidad, aquellos jóvenes que desean estudiar hasta el noveno grado deben viajar hasta el centro de Zacatecoluca en el Centro de Estudios de San Juan, la mayoría de jóvenes entrevistados dijeron asistir a esta escuela luego de terminar sexto grado. Este es uno de los motivos, entre otros, por el que el nivel de estudio entre los habitantes de la comunidad es tan bajo, tanto en adultos como en jóvenes. El dato común entre la población es haber estudiado entre primer y segundo ciclo (Gráfico $\mathrm{N}^{\circ} 5$ )

\section{Gráfico $\mathbf{N}^{\circ} 5$}

Nivel de estudio

\subsubsection{Ocupación}

En el gráfico $\mathrm{N}^{\circ} 6$, podemos observar los porcentajes de los habitantes de la comunidad en las diferentes ocupaciones. El $17 \%$ son agricultores en pequeño, que trabajan sus propiedades, pero al no ser rentables los cultivos a los que se dedican la mitad de la población trabajan como jornaleros, específicamente a la zafra de caña. 


\section{Gráfico 6}

\section{Ocupación}

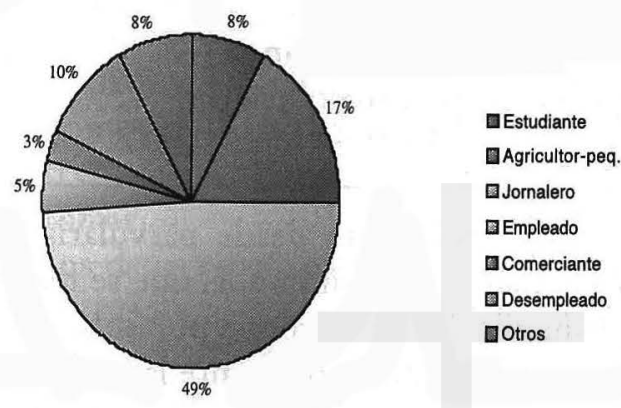

Existe además otro tipo de ocupaciones que representan porcentajes menores como: peluqueros, electricistas, artesanos, albañiles, sastres.

\subsubsection{Fuentes de Ingreso}

Como dijimos anteriormente, en el Gráfico $\mathrm{N}^{\circ} 7$ podemos observar que la mayor parte de la población se dedica a la agricultura en cuanto a zafra de caña, y la otra parte representativa lo conforman los

\section{Gráfico 7}

\section{Fuente de ingreso que posee} trabajadores individuales que tienen un oficio propio.

Es importante mencionar que una parte importante de la pobla-

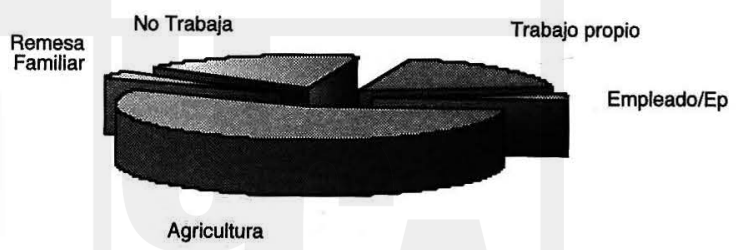
ción no trabaja y tan solo 2 familias viven de las remesas

\section{Modelo de atención institucional ${ }^{11}$}

En la comunidad San José las Flores, las ONGD desarrollan conjuntamente un modelo establecido por FUNDESA llamado "Modelo de Atención Institucional”. Este modelo es un instrumento para construir una estrategia que siente las bases para el desarrollo comunal rural, integrando elementos fundamentales que contribuyan al mejo- 
ramiento de la calidad de vida de la población. El modelo, según lo expresado por el representante de FUNDESA, Douglas Barahona, parte del "fortalecimiento de la familia, la comunidad rural y la participación equitativa de hombres y mujeres, en la construcción de procesos de trabajos orientados a impulsar el desarrollo local en el mediano y largo plazo".

El modelo integra tres programas estructurados en función de responder a las principales necesidades en las comunidades, para generar impactos positivos en la calidad de vida de las familias: programa de formación y organización, programa de desarrollo económico y medio ambiente y programa de seguridad habitacional.

\subsection{Programa de formación y organización}

El primer programa que se desarrolló en la comunidad fue el de formación y organización, propio de la visión de desarrollo de FUNDESA. Este programa contribuyó al fortalecimiento de la organización social y a la promoción de actividades de formación, "se generó en los líderes comunales una visión integral del desarrollo (social, comunal, sectorial y zonal) y capacidad de movilización e incidencia política a nivel local, municipal y nacional"12.

Los planes regionales de FUNDESA buscan fortalecer la organización y la capacidad de incidencia de las comunidades mediante procesos de acompañamiento a las mismas.

Este programa es importante que se realice como punto de partida, ya que sin la organización y la participación equitativa de hombres, mujeres y jóvenes, no es posible desarrollar los siguientes programas.

\subsubsection{Estrategias de formación y organización}

Las estrategias correspondientes al programa de formación y organización son las siguientes:

- Promoción y realización de actividades orientadas al rescate histórico, cultural y a la convivencia democrática.

- Formación y legalización de ADESCOS.

- Asesoría y acompañamiento a los procesos de planificación y gestión comunal. 
- Formación y capacitación de líderes y dirigentes comunales multiplicadores del conocimiento.

- Sistematización de experiencias educativas vinculadas a la práctica y a las vivencias del quehacer comunitario de los participantes.

La metodología para el desarrollo de las actividades educativas y formativas es la educación popular, priorizando en la aplicación del principio de aprender haciendo y de partir de la experiencia práctica y de las necesidades e intereses de los participantes. La educación popular reconoce que muchas estructuras económicas, políticas y educativas en el mundo son autoritarias, antidemocráticas y responde al interés de algunos grupos. La educación popular no da respuestas finales; más bien, hace preguntas y plantea problemas relacionados con la vida de los participantes, con el fin de fomentar la conciencia crítica.

\subsubsection{Organización}

Gracias al apoyo local de FUNDESA se ha logrado que la comunidad avance hacia procesos propios de desarrollo, fundamentados en una organización primaria y luego participativa de todos los habitantes. De esta forma, en la actualidad las estructuras organizativas de la comunidad San José las Flores, son:

\begin{tabular}{|l|l|l|}
\hline ADESCO & \multicolumn{1}{|c|}{ COMITÉS } & COOPERATIVAS \\
\hline $\begin{array}{l}\text { Junta Directiva } \\
\text { ADESCOSJF }\end{array}$ & $\begin{array}{c}\text { - Comité de Emergencia } \\
\text { y Medio Ambiente. } \\
\text { - Comité de Jóvenes. } \\
\text { Comité de Mujeres. } \\
\text { - Comité de Salud. }\end{array}$ & $\begin{array}{l}\text { Asociación Coopera- } \\
\text { tiva de Producción } \\
\text { Agropecuaria San José } \\
\text { las Flores. }\end{array}$ \\
\hline
\end{tabular}

La comunidad San José las Flores opera a través de una Junta Directiva constituida por once miembros. Si bien existe participación de mujeres, lo es en cargos calificados como "menos importantes". La Junta Directiva está integrada por cinco mujeres que fungen como tesorera, síndica y vocales; y por seis hombres que ocupan los dos cargos más importantes: presidente y vicepresidente. Los otros cuatro son vocales. 
La forma en que funciona esta Junta Directiva, es la siguiente: la Junta Directiva se reúne entre cada ocho y quince días, dependiendo de las necesidades. En la reunión, se tratan los problemas claves que sufre la comunidad y sus propuestas de solución. Esta decisión se comunica directamente a la Asamblea, que está conformada por todos los habitantes de la comunidad. Entre todos llegan a un acuerdo de la siguiente actividad a realizar en pro de la comunidad y la Asamblea debe decidir si acepta o no la propuesta de la Junta Directiva. Una vez tomada una decisión por parte de la Asamblea, la Junta Directiva lleva la propuesta a FUNDESA. Si es aprobada gestiona, un proyecto de financiamiento a través de Solidaridad Internacional Andalucía y/o Provida.

\subsubsection{Participación con otras comunidades}

En la comunidad San José las Flores se han promovido espacios de coordinación intercomunal y municipal, contribuyendo a generar discusiones sobre procesos de desarrollo con una visión de mediano y largo plazo a través de la planificación participativa, con líderes y representantes comunales.

Los intercambios de experiencias intercomunitarias, el desarrollo de convivios y actividades culturales, son de mucha importancia en el proceso de educación popular.

Para el tratamiento de problemas o necesidades que surgen a nivel de sector, las Juntas Directivas de las comunidades del sector se reúnen y comparten el problema, y los siguientes pasos se efectúan de la misma forma que si la necesidad ha tratar fuera solamente a nivel comunal, es decir, las decisiones se comparten y discuten con las Asambleas respectivas y una vez tomada una decisión se transmiten a FUNDESA.

Estos intercambios comunales se hacen con el fin de abrir un espacio para compartir puntos de vista de dirigentes comunales de distintos municipios del país, donde Fundesa esta implementando su trabajo.

\subsection{Programa de desarrollo económico y medio ambiente}

Este programa se ha realizado con la supervisión, propuestas y apoyo de las tres ONGD cooperantes con la comunidad. El progra- 
ma de Desarrollo Económico y Medio Ambiental, como su nombre lo indica, consiste en la construcción de sistemas productivos sostenibles ecológica y socialmente, enfatizados en la producción y seguridad alimentaria.

La implementación de este programa comenzó primero, con la selección conjunta de las familias con el potencial para desarrollar este proceso, esto por medio de FUNDESA y la Asamblea.

En la comunidad, existen, por un lado, las parcelas grandes que miden aproximadamente cuatro manzanas. Luego del proceso de selección y debido a la escasez de tierra, se repartieron solamente 46 parcelas.

Por otro lado algunos trabajan en pequeñas áreas de terreno de diez metros cuadrados en los solares, que justamente están ubicadas en la propiedad privada de las mismas. Este tipo de terreno ha sido proporcionado así con el fin de facilitar el cuido y la atención permanente de los cultivos, estas áreas son los llamados huertos caseros, que utilizan el método de la asociatividad de cultivos.

El programa está dirigido a la formación de fincas integrales, que consisten en la integración de diferentes rubros productivos de forma planificada y ordenada y permite desarrollar condiciones para la creación de modelos de agricultura sostenible, facilitando a las familias un mejor aprovechamiento de sus recursos. Esta actividad permite cosechar distintas variedades de frutas, producir en distintas épocas del año, contribuir a reforestar y por tanto mejorar el clima y el medio ambiente.

Los granos básicos han sido y seguirán siendo los soportes fundamentales de la dieta alimentaria de las familias rurales. En el caso específico del cultivo de los granos básicos las familias de la comunidad lo utilizan sobre todo para consumo. Existen también cultivos agroforestales y de hortalizas.

En la comunidad se producen 14 variedades diferentes de cultivos: berenjena, zanahoria, cilantro, variedades de frijol, arroz, maíz dulce, sandia, pipián, rábano, chile, remolacha, repollo, pepino y tomate.

En cada vivienda existen además mini granjas de producción familiar de aves, especialmente para el cuido de gallinas ponedoras, pero además hay quienes cuentan con cerdos, patos y otras especies que permite mejorar la calidad de la alimentación, tener ingresos y asegurar las condiciones de saneamiento ambiental. 
En segundo lugar, debido a que se trata de producción orgánica es necesario la capacitación y concientización de los habitantes, gradual y progresivamente para disminuir el uso de agroquímicos y aumentar la producción orgánica, para disminuir los daños que causan a la salud y al medio ambiente el uso indiscriminado de agroquímicos. En este aspecto la Asociación de ayuda humanitaria Provida ha contribuido con algunos seminarios también.

El método biointensivo es el que utilizan las familias para la producción orgánica. Este método incluye actividades como: doble excavación, asociación de cultivos, siembra cercana, producción de semilla, control biológico de plagas, elaboración y uso de productos orgánicos. Los beneficios de este método comienzan con la contribución directa de mejorar la textura y estructura del suelo, además de que no dependen de recursos externos para su mantenimiento, reduce los costos de producción y por supuesto no contamina el medio ambiente.

Dentro de estas capacitaciones es necesario tomar en cuenta los aspectos referentes a tecnología apropiada para la producción y sistemas de microriego. FUNDESA ha promovido en la comunidad el uso de tecnología apropiada con recursos locales sostenibles, de bajo costo y que generan buenos resultados para las fincas integrales

Entre las tecnologías empleadas se destacan las siguientes: la elaboración y el uso de productos orgánicos, pequeños sistemas de riego por goteo, obras de conservación de suelo, uso de semillas mejoradas, obras de captación de aguas lluvias, pequeñas represas instaladas en pequeños afluentes; y manejo de micro cuencas. Como observamos se ha priorizado el uso de tecnologías relacionadas con la conservación del recurso hídrico.

El programa de Desarrollo Económico y Medio Ambiente no puede reducirse a la producción para el consumo familiar, sino que debe incluir una producción extensiva para la comercialización para potenciar las habilidades y conocimientos de los productores que han demostrado interés y vocación para desarrollar el proceso productivo.

Esta fase busca ampliar el área y mejorar la calidad de la producción, y desarrollar mejores capacidades para la comercialización. Esta fase se orienta a fortalecer la planificación productiva; el acceso al mercado local, municipal y regional; el desarrollo de capacidades para 
la comercialización; la diversificación de la producción; la consolidación de grupos organizativos; y el uso de tecnología apropiada.

En la comunidad, el cultivo más rentable ahora es el pipián, ya que, debido a la baja en los precios de compra en el maíz, ya no es conveniente su producción. El pipián es ahora el producto que los habitantes de la comunidad utilizan mayoritariamente como fuente de ingresos. El pipián se produce en las parcelas de las familias y los productores lo venden a un solo habitante de la comunidad, quien lo comercia en el Mercado Central de San Salvador.

\subsection{Programa de Seguridad Habitacional}

Solidaridad Internacional Andalucía con la colaboración de su socio local (FUNDESA), puso en marcha un proyecto que inició con la construcción de 60 viviendas para familias que estaban en una situación de pérdida total de inmuebles, y continuó hasta totalizar 74 viviendas. La ejecución del proyecto se realizó formando grupos de trabajo dentro de los beneficiarios, bajo la dirección de un maestro de obra, para construir sus propias casas.

Solidaridad Internacional Andalucía propone una vivienda-tipo de Desarrollo Progresivo Modular. Por medio de esta concepción de la vivienda, los beneficiarios pueden participar del diseño de su propia casa, mediante métodos participativos. La dotación de viviendas en San José Las Flores por parte de Solidaridad Internacional asciende a un monto de 210.125 euros $^{13}$

La seguridad habitacional es un componente clave del Modelo de Atención Institucional debido a que proporciona estabilidad y seguridad, tanto por la construcción física de la vivienda como por las condiciones de saneamiento ambiental. Se trata de un componente social que vincula e integra a la población. El programa de seguridad habitacional consistía en la construcción de viviendas permanentes ${ }^{14} \mathrm{y}$ se ejecutó teniendo en cuenta normas de calidad de materiales, ubicación de los lotes y condiciones de la zona.

El programa incluyó la capacitación en albañilería de las familias participantes para que posteriormente puedan ampliar sus viviendas y/o que puedan trabajar como mano de obra semicalificada para otros proyectos o como fuente de generación de ingresos. En la comunidad 
en estudio únicamente 3 habitantes se dedican a prestar servicios remunerados de albañilería.

El programa de seguridad habitacional desarrolló tres contenidos: a) promoción y organización; b) construcción de viviendas; y c) módulo de agua y saneamiento.

En un principio se buscó que las familias más vulnerables fueran beneficiadas, organizando grupos de trabajo partiendo de la cercanía geográfica o de la afinidad entre familias. El equipo de promoción aseguró el buen funcionamiento del proceso constructivo en coordinación con el ingeniero residente, quien cercioró la calidad de la construcción. Es importante, resaltar el hecho que FUNDESA priorizó el acceso de la mujer jefa de familia a los proyectos de vivienda, con el fin de que ella y sus hijos gocen de sus múltiples beneficios, particularmente de la seguridad legal de la misma.

De este modo, existen ahora en total 74 viviendas donadas por Solidaridad Internacional en la comunidad que podrían ser descritas todas de la forma siguiente:

- Las viviendas incorporan medidas contra terremotos y un diseño anti-inundaciones.

- Un suelo encementado que se levanta sesenta centímetros del terreno natural mediante muros de bloque de hormigón.

- La pared construida de bloque mixto.

- El techo de teja romana.

Las diferencias entre las primeras sesenta viviendas construidas y las catorce restarntes son mínimas y reside en una mejoría en la calidad de los materiales utilizados. La diferencia más notoria es que las últimas diez casas construidas poseen una división adicional de la habitación. El lote de la vivienda en su conjunto es de 36 metros cuadrados. Según Solidaridad Internacional, "los materiales empleados en la construcción de las viviendas son permanentes, con una garantía de duración de cincuenta años y sirven para que una vez realizada una futura ampliación con recursos de los propios beneficiarios, toda la vivienda tenga un carácter homogéneo". ${ }^{15}$

Un hecho preocupante es que en la comunidad todos poseen vivienda propia por ser una donación, pero aún no poseen las escrituras, las están gestionando con el ISTA, proceso que ha sido bastante 
lento y que no parece tener una salida rápida, según argumentaron la mayoría de los habitantes de la comunidad.

\section{3.1 Iluminación y ventilación}

Cada uno de los espacios tiene regulares condiciones de iluminación y ventilación puesto que cuentan con 3 ventanas de $1.20 \times 0.80 \mathrm{~m}$ y la altura de cumbrera de la vivienda es de aproximadamente $3.00 \mathrm{~m}$, permitiendo así un fácil acceso del aire y de luz. Aunque podría haber sido mejorado con algún tipo de sistema de ventilación cruzada no está en tan malas condiciones.

\section{3.2 Número de espacios internos}

Las viviendas consideran la subdivisión del espacio interno en dos pequeñas habitaciones, ambos espacios están separados por una pared interna con un vano central de acceso y sus dimensiones son de $2.80 \mathrm{~ms}^{2}$ Como ya mencionamos, las diez viviendas que fueron construidas posteriormente cuentan con una división adicional de las habitaciones.

\section{3.3 Corredor}

La mayoría de viviendas han sido provistas de un corredor frontal, el cual es utilizado en muchas ocasiones como espacio de cocina o sitio de almacenaje de algunos utensilios domésticos. Estos corredores tienen un promedio de dos metros de ancho y se extienden a lo largo de toda la vivienda.

La presencia de los corredores se convierte en un extensor de la vivienda. Por otra parte, el corredor deriva en un área de extensión de las actividades propias de la familia, convirtiéndose en un área de juegos de los niños, área de estar, zona para el desarrollo de actividades manuales, etc.

La existencia del corredor permite además volver un poco más frescos los ambientes internos de la vivienda, puesto que le proporcionan un mayor espacio de sombra durante el día, así como una circulación permanente de aire fresco. 


\subsubsection{Cocina}

La tipología existente en la zona considera la construcción de un "espacio" para cocina ubicado al exterior de la vivienda, generalmente la cocina está ubicada en el corredor frontal que se adiciona a la casa.

Utilizan cocina de leña denominada "Finlandia ahorradora"; pero solamente existen cincuenta cocinas de este tipo en la comunidad. Cada cocina ahorradora de leña está compuesta por un comal de barro colocado sobre un agujero cubierto de cemento en el cual se coloca la leña, el humo sale por una tubería que va a dar arriba del techo. De esta forma se eliminó el problema de que el humo de la cocina se encerrara en la casa volviendo imposible la permanencia en su interior y la propensión a generar enfermedades respiratorias sobre todo en las mujeres que son las encargadas de la cocina.

Las estufas ahorradoras de leña son una tecnología sencilla que ha permitido el cuidado a la salud de las mujeres y niños, por la exposición constante a la combustión de la leña, con su consiguiente reducción de la incidencia de enfermedades respiratorias y oftálmicas. Además, los habitantes manifiestan que se han reducido los accidentes caseros producidos por el fogón tradicional o de piedras, en los miembros más pequeños de la familia.

La obtención de energía mediante madera vieja, los residuos de la cosecha, los cercos, y todo material que la proporcione, ahora se vuelve una tarea más difícil para los campesinos y campesinas. La leña continúa siendo la principal fuente de energía para la mayoría de los habitantes rurales pobres del mundo y también para muchos residentes urbanos. El gran consumo de leña como principal fuente energética representa un gran impacto a las zonas de vegetación natural, acahuales y zonas de vegetación compacta. Sumado a esto, la parcelación de las tierras y el desmonte de las zonas forestales han aumentado la escasez y restringido el acceso a la leña.

Por lo anterior, un aspecto importante de estas cocinas es la reducción en el consumo de leña, como su nombre bien lo indica, y por tanto, la reducción del impacto a las zonas forestales compactas. A esto se le suma la reducción de jornales en la búsqueda y corte de leña y la reducción de tiempo dedicado a cocinar, pues con este tipo de cocinas se pueden cocinar tres cosas al mismo tiempo 
Este tipo de cocina también ha permitido el mejoramiento a la vivienda, reduciendo el hollín al interior de la cocina

\section{3.5 Módulo de agua y saneamiento}

Al interior de todas las viviendas se encuentra un pozo de consumo familiar de $8 \mathrm{~m}$. de profundidad, el cual posee una bomba manual. Solamente en dos casas, las familias por cuenta propia han adquirido una bomba eléctrica cada una, a un costo de $\$ 100$.

En el año 2002, FUNDESA realizó un diagnóstico sobre la calidad del agua en comunidades del sur del municipio de Zacatecoluca, el cual "reafirmó que la mayoría de familias rurales viven en un estado permanente de precariedad, como consecuencia de la vulnerabilidad social acumulada por generaciones".

$\mathrm{El}$ análisis microbiológico, físico-químico y bacteriológico del agua de la comunidad San José las Flores, al igual que las comunidades de la zona, obtuvo un diagnóstico preocupante por presentar un alto grado de contaminación.

Debido a los resultados de la investigación sobre la contaminación del agua, desde el año 2001, la comunidad cuenta con dos pozos comunales de 26 y 54 metros de profundidad, lo cual ha contribuido a minimizar las enfermedades de esta fuente. De los cuales, en este momento solo el primero se encuentra habilitado. Los pozos tienen bombas manuales de mecate utilizadas para extraer agua del subsuelo.

La bomba manual ${ }^{16}$ tiene como elemento fundamental un mecate auto enlazado o "sinfín", por medio del cual y accionándolo en "circuito cerrado", hace posible mover hasta la superficie porciones continuas de agua. Estas bombas también son utilizadas con propósitos agrícolas o ganaderos, para el riego y el suministro de agua para animales.

Actualmente, una red hidráulica de siete cantareras se construye con mano de obra de la comunidad. Para este proyecto se ha repartido las 79 familias en siete grupos de cinco a seis personas cada uno, y tanto hombres como mujeres participan en jornadas de $8 \mathrm{AM}$ a $4 \mathrm{PM}$, con período de almuerzo y de descanso.

El sistema de servicio de agua potable será de uso domiciliar, lo cual implica que cada vivienda contará con su propio grifo y contador. 
Las ONGD cooperantes con la comunidad facilitaron la construcción de infraestructura para disminuir los niveles de contaminación del medio ambiente y los focos de reproducción de zancudos, tales como: pilas, baños y fosos resumideros de aguas grises.

\section{3.6 Letrinas}

El mecanismo de disposición de excretas que utiliza la población en el área rural, junto con los recursos hídricos, los hábitos higiénicos y la deposición de la basura tiemen una relación directa con el medio ambiente $y$, por consiguiente con la salud y la calidad de vida.

En la comunidad San José Las Flores, el 100\% de las viviendas dispone de letrinas aboneras, con lo cual aprovechan las excretas para fabricar abono orgánico ${ }^{17}$ de alta calidad libre de bacterias patógenas para el cultivo.

El tipo de letrinas en especifico es letrina seca abonera familiar (LSAF), que posee algunos beneficios a simple vista: no usa agua, no atrae moscas, ni produce mal olor.

La LASF consiste en una taza o asiento especial (con separación para heces y orina) y poseedora de dos cámaras recipientes o pozos que se usan en forma alterna; una se está llenando mientras la otra descompone el material previamente depositado. Cuando el primero está por llenarse el espacio libre se debe rellenar con tierra o cal, luego se sella y se empieza a usar el otro pozo. Cuando el segundo pozo está casi lleno, se debe vaciar el primero para volverlo a poner en uso. De esta forma, la destrucción de los organismos patógenos es garantizada luego de un año de sellado del pozo.

\section{Infraestructura social}

A pesar de que la comunidad cuenta con pocos recursos económicos, se han logrado cubrir ciertas prioridades, con el paso del tiempo. Las obras sociales de la comunidad a la fecha son las siguientes:

- Una iglesia evangélica

- Una cancha de fútbol: es la única área recreativa para los/as jóvenes y niños/as. Es importante aclarar, sin embargo que esta cancha forma parte del centro educativo, y que además se comparte con las comunidades aledañas. 
- Clínica médica: desde marzo de 2004, la comunidad cuenta con un Dispensario Médico. En la sección que habla de la salud de la comunidad, especificamos la situación de esta clínica.

- Casa comunal multiusos en construcción, dentro de la cual, además del salón multiusos, habrá una guardería infantil para la cual ya se posee el mobiliario y una oficina para reuniones de la Junta Directiva y Asamblea General.

- Red hidráulica con siete cantareras en construcción

- La iglesia católica está en construcción.

\section{Salud}

El estado de salud de la población es un dato inherente al desarrollo humano y que repercute en gran medida sobre el desarrollo integral de la comunidad. Evidentemente, una población sana y con una cobertura sanitaria eficaz y eficiente está en mejores condiciones para impulsar el desarrollo y para alcanzar un nivel de vida óptimo.

Anteriormente la salud de los habitantes era atendida solamente en el ámbito sectorial en La Hacienda Escuintla. En la actualidad, en el Centro Escolar de la comunidad San José Las Flores ya se cuenta con Calendarización de visitas médicas por parte del Ministerio de Salud en un promedio de una vez al mes.

Provida ha llevado a cabo programas de salud, como exámenes patológicos, campañas de desparasitación y vacunación, para la población en general.

Las enfermedades más comunes en la comunidad son: problemas en las vías respiratorias, infecciones urinarias, divieso, diarreas y dengue.

Desde marzo de 2004 gracias al apoyo de FUNDESA, Provida y Solidaridad Internacional, los habitantes de la comunidad cuentan con la Clínica Médica llamada "Dispensario Médico-Comunidad San José Las Flores", la construcción de esta clínica se llevo a cabo con la ayuda de mano de obra de la Comunidad el Papayal, porque los servicios se comparten con todo el sector.

El centro de salud atiende medicina general e imparte terapias respiratorias. Cuenta con poco equipo médico: báscula, instrumentos para pequeñas cirugías, una camilla y utensilios para terapia respiratoria. 
El dispensario médico como pudimos observar en la visita a la comunidad, solamente cubre la medicina básica y además no cuenta con apoyo especializado como doctores, enfermeras o estudiantes de medicina, más bien los mismos habitantes han formado un Comité de Salud con la ayuda y capacitación de Provida. Por ello, si se presenta alguna enfermedad grave o una cirugía mayor deben acudir a Zacatecoluca, adonde además solo existe un hospital y una unidad de salud.

Provida también ha impartido capacitaciones sobre: género acerca de los problemas intra familiares, tratamiento de los huertos caseros y abono orgánico, utilización eficiente de la leña e higiene, capacitación sobre letrinas, tratamiento de aguas, entre otras.

\section{Otros servicios básicos:}

\section{Energía eléctrica}

Las 79 familias cuentan con el servicio de energía eléctrica y hacen uso a nivel domiciliar. La empresa eléctrica que suministra el servicio a todos es la Distribuidora Eléctrica del Sur S.A. (DELSUR). Dado que la comunidad está bastante aislada, el hecho que posean electricidad es muy importante para satisfacer las necesidades básicas. En la mayoría de casas solamente cuentan con algunos aparatos eléctricos como caseteras, radios, televisores y refrigeradoras

Cuentan además con alumbrado público proporcionado y financiado por parte de la Alcaldía de Zacatecoluca.

\section{Medios de transporte}

En la comunidad solamente existe un bus que realiza dos viajes al día, de San José Las Flores a Zacatecoluca y viceversa, el primer viaje es al mediodía y el segundo en la tarde. Este bus es propiedad privada de un habitante de Zacatecoluca que ha decidido brindar su transporte en esta comunidad y las comunidades aledañas. El valor del transporte por persona es de $\$ 0.60$.

El sector del transporte y las comunicaciones, tanto las vías de comunicación como los medios de comunicación, son de vital importancia para el desarrollo económico. La zona en que está ubicada San 
José Las Flores está bastante aislada y su acceso es difícil. No llegan los vehículos estándar, solamente pick-up o vehículos grandes.

Debido a que dentro de la comunidad existen solamente 5 pickups de propiedad privada, el medio de transporte utilizado por los habitantes son las bicicletas, todas las familias poseen al menos una, debido a su bajo costo y practicidad.

\section{Medios de comunicación}

Desgraciadamente la comunidad no cuenta con servicio de teléfonos públicos y mucho menos teléfonos fijos, lo cual empeora su situación de aislamiento, por su lejanía con la ciudad. Según nuestro estudio, solamente de dos a cinco personas cuentan con teléfono celular. Existen también dos teléfonos eléctricos de tarjeta de propiedad privada.

\section{Listado de problemas priorizados}

\subsection{Perspectivas de los habitantes de la situación actual}

Según nuestra visita a la comunidad, la mayoría de los habitantes manifiestan encontrarse económicamente mejor que hace cinco años. Esta idea tiene mucho que ver con los programas realizados por las ONG cooperantes, principalmente por FUNDESA, debido a que desde el huracán Mitch, es decir desde 1998, están trabajando conjuntamente con la comunidad. Sin embargo, el $16 \%$ restante piensa que se han mantenido igual, ya que los avances en su bienestar se contrarrestan con la situación económica en general de El Salvador.

\section{Gráfico $\mathrm{N}^{\circ} 8$}

¿Cómo considera que ha estado su sistema económico en los últimos 5 años?

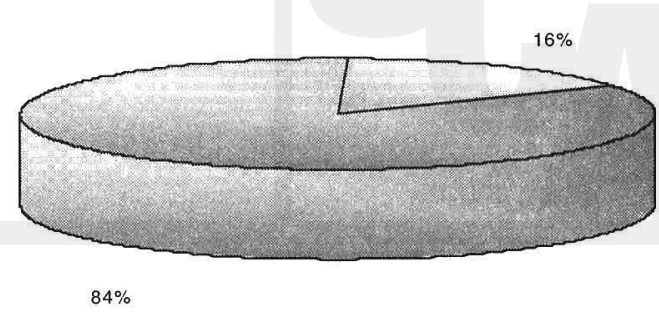

巴Se ha mantenido igual $\square \mathrm{Ha}$ mejorado económicamente 
Ha pesar de haber mejorado la situación de bienestar de los habitantes de la comunidad, siguen existiendo algunos problemas.

Los problemas principales que presenta la Comunidad San José làs Flores son la reparación de calles y el servicio de transporte. En cuanto al primero, nos referimos a la reparación al menos de las calles principales de la comunidad para evitar las inundaciones en épocas de lluvia. Esto implica adoquinar y construcción de cunetas en las calles internas. El problema de reparación de calles se ve reflejado también en la ausencia de servicio público de transporte, debido a las dificultades de acceso a la comunidad. Si bien existe un bus que hace los viajes de Zacatecoluca a la comunidad y viceversa, la tarifa de este bus es alta, y la frecuencia de los viajes es poca.

Además de estos problemas podemos encontrar muchos más en las diferentes áreas sociales

Estos mismos problemas se traducen en necesidades que deben ser atendidas a la brevedad posible, pero debido a que los proyectos implementados por las Organizaciones voluntarias han sido enfocados en prioridad al cubrimiento de necesidades básicas, la resolución de estos problemas se ha ido postergando.

En cuanto a los recursos naturales y prevención del riesgo, tenemos: la construcción de bordas y puente en el río Amayo, la obra de paso en la rampa cañada Ausingo y la reforestación de zonas verdes.

En el área social es necesario: ampliación de escuela, finalizar construcción de casa comunal multiusos y guardería infantil, seguridad de Policía Nacional Civil.

En cuanto a cultura y deporte encontramos las siguientes necesidades: crear un parque infantil o ampliar las zonas de recreación, celebrar un día festivo de la comunidad, fundar una biblioteca comunal, así como dotar de tela ciclón y mallas para la cancha de fútbol.

\section{Impresiones}

Un aspecto interesante fue conocer la forma en que los residentes de la comunidad San José las Flores están organizados para la realización de proyectos en pro de los mismos. Se organizan de tal forma que todos participan en el desarrollo de proyectos conformando gru- 
pos de trabajo que incluyen hombres y mujeres para un trabajo duro como la construcción. En este ámbito, una variante a resaltar es el buen funcionamiento de la Junta Directiva que vela por el bienestar de la comunidad. Además, fue creada democráticamente y cuenta con la participación tanto de hombres como de mujeres y jóvenes.

Las técnicas para el tratamiento de la tierra que permiten una producción de catorce variedades de cultivo. La principal técnica es la de abono orgánico conocido como vinagre de madera, la cual consiste en crear un hoyo de $1.5 \mathrm{mts}$. de profundidad donde se coloca madera seca de pito, madrecacao y otra madera leguminosa que se deja fermentar, que por medio de un caño se lleva hasta los cultivos sirviendo como abono. Esta técnica como muchas otras nos permite darnos cuenta que a falta de recursos económicos para comprar un abono químico, que además de caro es contaminante, los medios que la comunidad utiliza para sacar adelante la producción de cultivos además de sencilla es en beneficio del medio ambiente.

Otro punto que habla muy bien de la comunidad, es el diseño de las cocinas que permiten el ahorro de leña. Ésta consiste en un horno con dos o tres orificios y una chimenea delgada que sale por el techo de la vivienda. La colocación de la leña es dentro de la cocina y en cada orificio se colocan utensilios de cocina que permiten cocinar varias comidas a la vez, reduciendo así el gasto de leña, que como ya mencionamos es bastante escasa en estos tiempos.

Entre los aspectos que más nos llamaron la atención, se encuentran parte de los proyectos realizados con el apoyo local de FUNDESA, en específico aquellos que mejoran la calidad de vida humana:

- Los pozos de los cuales se obtiene agua.

- La cocina ahorradora de leña y los beneficios para cada familia, tal y como se describió anteriormente.

- La agricultura orgánica que se desarrolla en la comunidad, es parte esencial de los procesos de producción.

- La integridad de género que existe en la comunidad con mayor participación de la mujer en todas las actividades, a pesar de ser una comunidad que apenas está sentando los inicios del desarrollo de economía popular solidaria. 
Desde nuestro punto de vista, un problema generalizado en la comunidad, es el de la comercialización de la producción agrícola, ya que no hay organización de grupos asociativos para la producción ni financiamiento para la misma.

\section{Conclusiones}

La investigación de campo realizada en la comunidad San José las Flores nos permitió conocer la realidad sobre las economías populares, mostrándonos que esta comunidad posee una estrategia alternativa de y para las mayorías populares, enfocada en una visión económica, como también en muchos aspectos sociales que son parte de la comunidad. Por otra parte, es una estrategia fundadora de vida, porque, además de mejorar el bienestar, preserva el medio ambiente, bajo sus técnicas de cultivo orgánico y tratamiento orgánico de las tierras con ayuda de ONGD.

En la comunidad San José Las Flores ha habido un desarrollo social, entendido como el desarrollo de las capacidades humanas, el aprendizaje de los modos de hacer las cosas, los conocimientos necesarios para organizar y gestionar los procesos y actividades, la organización eficiente de las actividades, por parte de los sujetos que utiliza los recursos sociales disponibles. Y no sólo como obtención de cosas y bienes materiales que pueden satisfacer transitoriamente sus necesidades. La economía popular no supone tanto la satisfacción de las necesidades, sino el desarrollo de las capacidades propias para hacer frente a necesidades recurrentes y en expansión.

La economía popular supone la organización, la solidaridad y el esfuerzo activo de los mismos grupos y comunidades que lo experimentan. Un pueblo desorganizado no podrá jamás salir de la pobreza. Esto lo hemos constatado en la comunidad en estudio, que ha logrado encaminarse hacia un proceso de desarrollo por medio de la participación activa de los miembros de la comunidad, movilizando sus propias capacidades y esfuerzos para alcanzarlo, porque estimula las iniciativas, hace descubrir recursos y capacidades ocultas existentes en las personas y grupos, refuerza la voluntad, activa la conciencia, y da lugar a la formulación y puesta en marcha de proyectos que movilizan esas mismas capacidades y recursos.

Una experiencia de economía solidaria 
Mediante la educación popular y la capacitación se desarrolla la autoestima, se toma conciencia de los propios problemas o conflictos y de las energías disponibles para enfrentarlos; se expande el conocimiento de la realidad y de las propias capacidades y recursos; se perfecciona la información sobre las condiciones en que se desenvuelve la acción; se desarrolla la capacidad de tomar decisiones y de gestionar con eficiencia los recursos disponibles; se facilita, en general, un proceso de crecimiento personal y comunitario que es parte esencial y a la vez condición necesaria del desarrollo social.

Educación popular y economía popular, convergentes en los objetivos del desarrollo social, se necesitan y potencian mutuamente. Separadas y sin vincularse estrechamente, reducen su eficacia promocional. Por ejemplo: para que la producción de los cultivos sea eficiente han necesitado capacitaciones sobre técnicas de riego, asociatividad de cultivos, prácticas orgánicas, entre otros.

La superación de la pobreza y el desarrollo social son responsabilidad de toda la sociedad, las magnitudes de la pobreza son tales que de un modo u otro afectan a toda la población. Definitivamente, si en una sociedad hay muchos pobres, toda la sociedad es pobre y subdesarrollada. Si el problema es de todos, la superación de la pobreza y el desarrollo social son también responsabilidad de todos. En todo caso, el problema es tan amplio y agudo que sin la cooperación y solidaridad de todos no será posible resolverlo.

$\mathrm{Si}$ bien es cierto que la pobreza es responsabilidad de todos, el agente principal del desarrollo social y de la superación de la pobreza son las propias comunidades y grupos pobres afectados, en este caso las propias economías populares. La acción asistencial de ONG y otros grupos es necesaria en las economías populares, que generalmente carecen del apoyo del gobierno; claro está que no nos referimos al asistencialismo.

Esta comunidad refleja avances en aspectos económicos, sociales y culturales gracias a la ayuda internacional y de otras ONG locales involucradas con la comunidad como FUNDESA y Provida. En este proceso los objetivos de las acciones y proyectos de desarrollo social 
son definidos por las mismas comunidades. Los medios para el desarrollo social son puestos a su disposición, de modo que pueden gestionarlos autónomamente. La ejecución de las acciones ha de ser igualmente responsabilidad de los beneficiarios, quienes evalúan sus resultados conforme a propios criterios de costo-beneficios.

En este sentido, las múltiples y heterogéneas experiencias de la economía popular, sean individuales, familiares o colectivas, pueden considerarse como el más genuino comienzo del desarrollo social y de la superación de la pobreza.

La poca obtención de ingresos bajo la modalidad de trabajo comunitario es debido a la insuficiencia de empleo; sin embargo de no existir ésta modalidad los ingresos serían nulos.

Un aspecto importante de la comunidad es la producción fundamentada en agricultura orgánica. A diferencia de una economía capitalista que reviste una modalidad de producción industrializada que es degradante del medio ambiente, este tipo de producción lo preserva, pues se emplean técnicas de carácter orgánico, como el abono basado en el vinagre de madera (característica de esta comunidad) que es origen de un proceso netamente natural, pues es proveniente de la savia de árboles y utilizada para el tratamiento de la tierra, con el objetivo de no deteriorar el medio ambiente con productos químicos. Existe por tanto una mayor conciencia sobre problemas ambientales.

Consideramos que esta estrategia de desarrollo la economía popular solidaria es fundamental y puede existir y progresar, es pues además de permitir y mejorar el bienestar de la comunidad, permite preservar el medio ambiente y mantener las necesidades de la generación presente sin atentar con la capacidad de la generación futura para desarrollarse. Por lo tanto, puede constituir una estrategia alternativa de y para las mayorías populares con la cual se busca resolver problemas de pobreza y exclusión. Debido a esto no debe aislarse de la realidad del mundo capitalista en el que vive, sino más bien debe encontrar los mecanismos que le permitan interactuar como economía popular con las otras economías y aprovechar los beneficios de esta relación.

De acuerdo a la historia, es importante recordar que el desarrollo social es un proceso lento, que puede ser acelerado mediante proyec- 
tos y programas de largo plazo. Para estas economías populares, la pobreza no es una situación transitoria, sino un estado en el que se ha nacido o en el que se ha permanecido durante un largo período de la vida. Salir de este estado no puede ser sino el resultado de esfuerzos largamente sostenidos en el tiempo. Incluso para quienes han caído en la pobreza en forma más o menos repentina, superarla se convierte en tarea de años, porque la pobreza succiona a quienes caen en ella. Nadie puede pretender alcanzar el desarrollo social de los pobres mediante acciones puntuales y proyectos de corto plazo.

En este sentido, si bien las acciones de emergencia pueden ser necesarias para enfrentar situaciones coyunturales extremas, el desarrollo social requiere programas que se sostengan en el tiempo, durante años y décadas. El modelo de programas implementados por FUNDESA principalmente, que es quien posee mayor contacto directo con la comunidad, está dirigido a resultados de largo plazo, y podemos observar que en los casi 4 años que lleva acompañando a la comunidad, ésta ha tenido una gran mejoría en términos organizativos, de producción y obtención de servicios básicos.

\section{BIBLIOGRAFÍA}

Ortiz Roca, Humberto. Conferencia sobre la humanización de la economía global. http://wwrw.gresp.org.pe/humanieco.htm

Coraggio, José Luis. La agenda del desarrollo local. Ponencia presentada en el Seminario sobre "Desarrollo local, democracia y ciudadanía", Centro de Participación Popular (CPP), 3-6 de julio de 1996, Montevideo.

Razeto M., Luiz. “Cinco constataciones sobre la pobreza, catorce tesis sobre el desarrollo social, y una conclusión sobre la economía solidaria" wwweconomiasolidaria.cl

Medina, Regina, Programa de vulnerabilidad del bajo Lempa. Prevención y mitigación de desastres naturales. Estudio sobre vivienda, asentamientos bumanos e infraestructura. Proyecto MARN-BID. Noviembre de 2000.

Montoya, Aquiles, Desarrollo económico. Ed. Críticos, 2a. Edición, San Salvador, 2003

Solidaridad Internacional de Andalucía, Campaña sobre la vulnerabilidad social en Centroamérica 2003-2004: Centroamérica: ¿Desastres 
Desastres.doc

"Economía del Trabajo: una alternativa racional a la incertidumbre", Trabajo presentado en el panel Fronteras de la Teoría Urbana: CGE, Incertidumbre y Economía Popular, Seminario Internacional sobre Economía y Espacio, organizado por el Centro de Desarrollo y Planificación Regional (Cedeplar), Belo Horizonte, Brasil, 6-7 Diciembre 2001

Memoria 2002. Solidaridad Internacional.

Memoria 2001 FUNDESA

www.fundesa.org.sv

www.solidaridadinternacional.org

www.carecen.org.sv

www.chf.org.sv

\section{Notas}

1. El presente trabajo fue realizado en el curso de Desarrollo Económico, impartido por Aquiles Montoya.

2. Según el texto "Economia del Trabajo: una alternativa racional a la incertidumbre", Trabajo presentado en el panel Fronteras de la Teoría Urbana: CGE, Incertidumbre y Economía Popular, Seminario Internacional sobre Economía y Espacio, organizado por el Centro de Desarrollo y Planificación Regional (Cedeplar), Belo Horizonte, Brasil, 6-7 de diciembre de 2001.

3. Análisis a partir del texto de Humberto Ortiz Roca, presidente del GRESP (Grupo red de Economía solidaria Perú). Conferencia sobre la humanización de la economía global.. http://www.gresp.org.pe/humanieco.htm

4. Ver Anexo 3 para más detalle

5. Basado en Economía del Trabajo: una alternativa racional a la incertidumbre.

6. Aquí se entiende por "hogar" el grupo que comparte y utiliza en común un presupuesto para la alimentación, la vivienda y otros gastos básicos

7. Los micro emprendimientos mercantiles son organizaciones colectivas de trabajo dirigidas a producir o comercializar bienes o servicios en los mercados. Pueden incluir miembros de la UD (familiares o no) así como otros trabajadores asociados o contratados. Su locus puede ser parte de la misma vivienda o un local aparte.

8. Análisis basado en el texto de Luiz Razeto M., "Cinco constataciones sobre la pobreza, catorce tesis sobre el desarrollo social, y una conclusión sobre la economía solidaria" wwreconomiasolidaria.cl

9. Análisis basado en el texto de José Luis Coraggio. La agenda del desarrollo local Ponencia presentada en el Seminario sobre "Desarrollo local, democracia y ciudadanía", Centro de Participación Popular (CPP), 3-6 de julio de 1996, Montevideo, Uruguay. bttp://www.chasque.apc.org/guifont/coraggio.btm 
10. Ver Anexo 7 para el formato de las encuestas y guías entrevista.

11. Cabe mencionar que este apartado está elaborado de acuerdo a los informes proporcinados por FUNDESA en cuanto a objetivos, misión y visión del modelo de atención institucional que propone FUNDESA, sin embargo la evolución del proyecto está enfocado de acuerdo a nuestra perspectiva y a los resultados obtenidos.

12. Tomado de la perspectiva del Modelo de Atención Institucional que desarrolla FUNDESA. www.fundesa.org

13. Memoria 2002. Solidaridad Internacional. http://www.solidaridad.org/pages/ frame06.htm

14. Ver Anexo 1 para más detalle de la construcción de las viviendas

15. Campaña sobre la vulnerabilidad social en Centroamérica 2003-2004: Centroamérica: ¿Desastres naturales o vulnerabilidad social? Solidaridad Internacional de Andalucía www.solidaridad.org/word/Desastres.doc

16. Ver Anexo 2, para una descripción más detallada del funcionamiento de las bombas de mecate manual.

17. Abono orgánico: Materia orgánica descompuesta (en putrefacción), normalmente de origen vegetal. Se aplica al suelo para incrementar su contenido en humus. 